\title{
EVALUACIÓN DE FACTORES DE RIESGO DE CARNE PÁLIDA, SUAVE Y EXUDATIVA (PSE) DEBIDO A LAS CONDICIONES PRESACRIFICIO EN CERDOS
}

\section{RESUMEN}

El objetivo del estudio fue determinar el efecto del manejo presacrificio porcino sobre la incidencia de carne pálida, suave y exudativa (PSE) y describir los factores de riesgo. Se recolectaron un total de 20 variables relacionadas con el transporte y las condiciones presacrificio durante un período de tres meses. Se estudiaron 134 lotes ( 3.156 cerdos) en una planta de beneficio comercial colombiana. Se usó una regresión logística multivariada para identificar los factores de riesgo previos al sacrificio. La calidad de la carne fue descrita como una variable de respuesta binomial (1: carne normal, 2: condición PSE). La incidencia de carne PSE fue 5,8\%. Los resultados indican que el peso, el tiempo de estadía en la planta de beneficio y la presencia de contusiones cutáneas incrementan el riesgo de presentación de la condición PSE $(p<0,05)$. El tiempo de reposo en planta entre 12,1 y $36 \mathrm{~h}$ incrementó de 4,3 hasta 9,9 veces la incidencia de la condición PSE comparado con tiempos de estadía en planta entre 4 y $12 \mathrm{~h}$. Además, el peso de los cerdos es un factor de riesgo que incrementó la incidencia de la condición PSE ( $p$ $<0,05)$. Sin embargo, el tiempo de transporte no estuvo relacionado con la presencia de valores de $\mathrm{pH}$ bajos.

Palabras clave: calidad de la carne, cerdos, estadía en la planta, estrés.

\section{EVALUATION OF RISK FACTORS OF PALE, SOFT AND EXUDATIVE (PSE) MEAT DUE TO PRE- SLAUGHTER CONDITIONS IN PIGS}

\section{ABSTRACT}

The goal of this study was to determine the effect of pigs' pre-slaughter handling on incidence of pale soft exudative (PSE) meat and describe risk factors. A total of 20 variables related to transport and preslaughter conditions were recorded over a three months period. A total of 134 lots (3,156 pigs) from a Colombian commercial slaughterhouse were studied. A multivariate logistic regression was used to identify the risk factors prior to slaughter. The pork quality was described as a binomial response (1: normal meat, 2: PSE condition). The incidence of PSE was 5.8\%. The results indicate that weight, time of stay in the slaughter house and the presence of skin bruising increase the risk of PSE condition $(p<0.05)$. A downtime from 12.1 to 36 hours at the slaughterhouse increased the incidence of PSE condition from 4.3 to 9.9 times compared to downtime periods between 4 and 12 hours. Besides, the weight of pigs is a risk factor which increased the incidence of PSE condition $(p<0.05)$. However, the transport time was not related to low $\mathrm{pH}$ values.

Key words: downtime, meat quality, pigs, stress.

\footnotetext{
1 PhD Universidad de Caldas, Facultad de Ciencias Agropecuarias, Programa de Medicina Veterinaria y Zootecnia, Departamento de Salud Animal; Grupo CIENVET. Manizales, Colombia. Correo electrónico: marlyn.romero@ucaldas. edu.co

${ }_{2}^{2}$ CPhD. Universidad de Caldas, Facultad de Ciencias Agropecuarias, Programa de Medicina Veterinaria y Zootecnia, Departamento de Salud Animal; Grupo CIENVET. Manizales, Colombia. Correo electrónico: jorge.sanchez@ucaldas. edu.co
} 


\section{INTRODUCCIÓN}

La cadena cárnica porcina colombiana ha orientado sus estrategias de competitividad hacia el cumplimiento de las expectativas del consumidor en cuanto a la calidad por medio de la diversificación de la producción, mejoramiento de la crianza y el sacrificio, al tiempo que se apoya en la investigación aplicada en temáticas que conlleven al aumento de valor agregado del producto final (1). La agrocadena ha definido como temas prioritarios la inocuidad, el control de enfermedades de presentación epidémica (como la peste porcina clásica), el fortalecimiento gremial, la capacitación y el fomento al consumo (1). Sin embargo, existe poca preocupación en la evaluación de los defectos de calidad de la carne porcina relacionados con las condiciones PSE y DFD (Pale, Soft, Exudative y Dark, Firm, Dry, por sus siglas en inglés, respectivamente), a pesar de que generan pérdidas importantes para la industria (2). Las condiciones PSE y DFD son limitantes para la producción de derivados cárnicos, porque no cumplen con las especificaciones técnicas requeridas para el procesamiento, disminuyen el rendimiento en canal, presentan menor aceptabilidad por parte del consumidor y limitan su comercialización $(2,3)$. En Colombia se han descrito prevalencias de condiciones DFD y PSE del 22,2\% y 25,2\%, respectivamente (2). Se considera que las pérdidas generadas por la condición PSE se encuentran entre los US \$20 y \$32 millones anuales, en países como Estados Unidos y el Reino Unido (4).

Las prácticas cruentas usadas durante el manejo de los cerdos tienen implicaciones éticas, económicas y sobre la calidad de la carne. Las canales provenientes de cerdos manejados inapropiadamente, presentan una mayor proporción de fracturas, hematomas, hemorragias petequiales y equimóticas, que conllevan a un aumento en los decomisos durante la inspección sanitaria, disminución del valor comercial de la carne y mayor mano de obra para el procesamiento $(5,6)$. De otra parte, estas prácticas son factores que favorecen la presencia de estrés agudo en los cerdos, inmediatamente antes del sacrificio, lo que causa un incremento en la tasa de acidificación postmortem y, por tanto, la presencia de carne PSE. En este contexto, es relevante establecer si las condiciones del presacrificio bajo lineamientos comerciales, generan estrés y afectan la calidad de la carne.

El objetivo del presente trabajo consistió en evaluar los factores de riesgo para la aparición de carne PSE relacionados con el manejo presacrificio en una planta de beneficio comercial localizada en Antioquia, principal zona porcícola de Colombia. Esta información puede ser útil para proponer cambios en etapas específicas del presacrificio, a fin de plantear mediciones sencillas y prácticas, para ser implementadas en las plantas comerciales.

\section{MATERIALES Y MÉTODOS}

\section{Descripción del estudio}

Se realizó un estudio de corte transversal durante los meses julio, agosto y septiembre de 2012 en una planta de beneficio localizada en el departamento de Antioquia, que cumplía con los requerimientos sanitarios exigidos por la legislación colombiana (7-9). Se estudiaron 3.156 animales (134 lotes) manejados bajo condiciones comerciales. Se evaluaron las buenas prácticas de transporte exigidas por la legislación colombiana (9), mediante la aplicación de un instrumento estructurado dirigido a los conductores $(\mathrm{n}=$ 71) y la observación directa de los vehículos $(n=134)$. Las variables estudiadas fueron: densidad en el camión $\left(\mathrm{kg} / \mathrm{m}^{2}\right)$, disponibilidad de espacio en el camión $\left(\mathrm{m}^{2} / \mathrm{cerdo}\right)$, peso promedio $(\mathrm{kg})$, velocidad promedio $(\mathrm{km} / \mathrm{h})$, tiempo de transporte $(\mathrm{h})$, densidad en el corral $\left(\mathrm{kg} / \mathrm{m}^{2}\right)$, disponibilidad de espacio en el corral $\left(\mathrm{m}^{2} /\right.$ animal), duración de la estadía en la planta de beneficio (h), tiempo de ayuno en la granja (h), tiempo de ayuno total (tiempo de ayuno 
en la granja + duración del viaje + tiempo de estadía en la planta sin alimento) (h), tipo de camión (uno y dos pisos), hidratación durante el viaje (sí, no), mezcla de animales en la granja (sí, no), inspección de los cerdos durante el viaje (sí, no), paradas durante el viaje para consumir alimentos (sí, no) y duración de las paradas (min).

\section{Condiciones de transporte y en la planta de beneficio}

En las granjas se seleccionaron los cerdos de diferentes corrales para garantizar la homogenidad de los lotes a transportar. Los cerdos se movilizaron en camiones de dos ejes con chasis rígido (combinado con madera y acero), provistos de ventilación pasiva, techo con carpa de lona y con una capacidad para 5 y 10 ton. Algunos vehículos estaban adaptados con dos pisos en su área de carga para el alojamiento de los porcinos. A la llegada de los cerdos a la planta de beneficio fueron descargados por medio de una rampa neumática, pesados y alojados en corrales de recepción techados y de paredes compactas, protegidos de las condiciones climáticas y provistos de suministro de agua fresca a voluntad, conservando el mismo grupo social proveniente de la granja. Los cerdos fueron conducidos en lotes a través de un corredor de paredes sólidas y fueron alojados en corrales individuales por lote. $\mathrm{La}$ insensibilización se hizo en una cámara de $\mathrm{CO}_{2}$ automática en donde eran localizados dos o tres animales, a continuación fueron izados y sangrados (Figura 1).

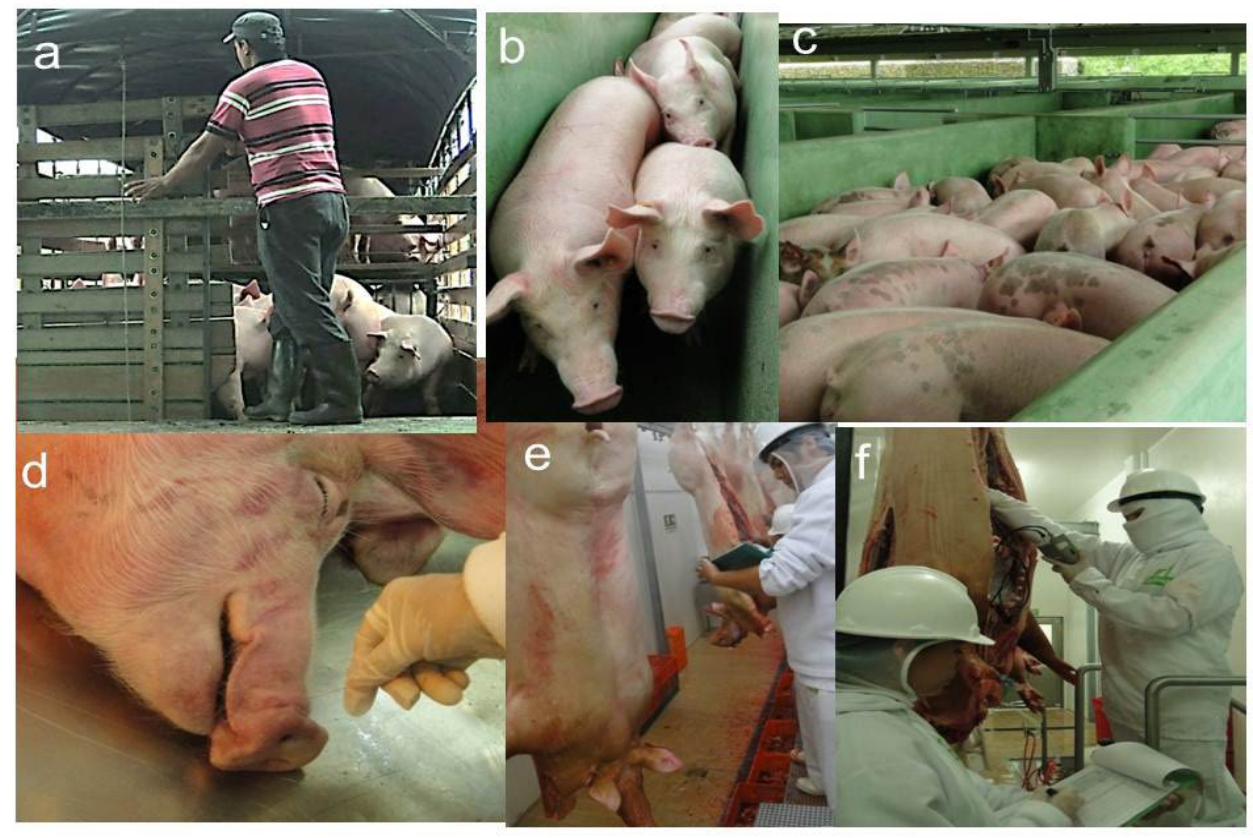

Figura 1. Características de la planta evaluada: camión típico para el transporte de porcinos en Colombia (a), conducción de los cerdos al área de descanso (b), corral de recepción techado (c), evaluación de la efectividad de la insensibilización (d), registro de contusiones cutáneas en las canales (e) y medición del $\mathrm{pH}_{45}$ (f). 


\section{Evaluación de las contusiones}

Las contusiones cutáneas se caracterizaron en la línea del proceso mediante la observación de las dos caras de la canal, procedimiento que fue realizado por un observador entrenado. Se estableció inicialmente la presencia o ausencia de contusiones cutáneas. A continuación se clasificaron según la localización topográfica (cabeza, espalda, medio, lomo y jamón), el tamaño, la concentración (agrupadas en un área específica de la canal o aisladas en partes separadas de la misma), el color (rojas y marrones), la forma (coma, rectangular, lineal, difusa y romboide) y tamaño (categoría 1: entre 0,5-1 cm, categoría 2: 2-5 cm, categoría 3: 6-10 $\mathrm{cm}$, categoría 4: $11-15 \mathrm{~cm}$ y categoría $5:>15 \mathrm{~cm}$ ) $(10,11)$.

\section{Evaluación de la efectividad de la insensibilización}

La efectividad de la insensibilización se estableció teniendo en cuenta la presencia de signos indicativos de la recuperación de la consciencia de los animales, en el intervalo de tiempo desde la salida de los cerdos del tunel de $\mathrm{CO}_{2}$ hasta el inicio de la sangría. Los signos observados fueron respuesta al dolor, por medio de la aplicación de una aguja en el septum nasal (Figura 1), reflejo palpebral, intentos de incorporarse y las vocalizaciones. La insensibilización se consideró efectiva cuando se presentó la ausencia de estos signos.

\section{Evaluación del pH}

Se efectuó la medición del $\mathrm{pH}\left(\mathrm{pH}_{45}\right)$ en el músculo longissimus thoracis (LT) entre la $3^{\mathrm{a}} \mathrm{y} 4^{\mathrm{a}}$ últimas costillas, en el lado derecho a $8 \mathrm{~cm}$ de la línea media dorsal, con un potenciómetro de punzón (IQ150 pH/Mv/medidor de temperatura; IQ Scientific Intruments). El equipo fue calibrado cada cinco muestras, usando dos soluciones estándar de pH 4 y 7; se efectúo lavado con agua destilada después de cada medición. Los criterios para definir la calidad de la carne fueron: carne PSE $\mathrm{pH}_{45} \leq 5,8$ y carnes normales $\mathrm{pH}_{45}>5,8$ (12).

\section{Análisis estadístico}

El análisis se efectúo utilizando el programa Stata Versión 12.0 (College Station, Texas, EU). La variable contusiones fue recodificada con valores de 1 (daño leve), que agrupaba las canales que presentaron entre 1 y 5 contusiones y 2 (daño severo) cuando se observaron entre 6 y 9 lesiones/canal. Los eventos observados durante la insensibilización fueron presentados como proporciones y las diferencias por medio del coeficiente de Pearson. Para evaluar el $\mathrm{pH}$, se realizó un análisis de regresión logística multivariada que asumió como variable de respuesta binomial el $\mathrm{pH}_{45}$, donde 0 correspondió a las carnes con $\mathrm{pH}_{45} \leq 5,8$ consideradas como carnes PSE y 1 aquellas con $\mathrm{pH}_{45}>5,8$, como carnes normales. El modelo general fue:

$$
Y=\frac{e^{\beta o+\sum ß i * ß X i}}{1+e^{\beta o+\sum ß i * ß X i}}
$$

donde $Y$ es la probabilidad de presencia de carne con $\mathrm{pH}_{45} \leq 5,8 ; \beta$ o el intercepto, $\beta$ i los coeficientes de correlación y Xi el vector de las variables independientes incluidas en el análisis.

Se realizaron análisis individuales para cada variable predictora, para explorar la información. A continuación, se ejecutó el modelo completo con las variables predictoras seleccionadas, para estimar sus efectos y grado de significancia. Las variables que no presentaron significancia fueron removidas una por una del modelo, comenzando por aquellas que presentaron un valor de $p>0,05$. Los modelos se volvieron a ejecutar para identificar las variables de confusión, por medio de la comparación de las estimaciones del nuevo modelo, con las estimaciones del modelo anterior. Finalmente, se realizó la prueba de bondad de ajuste del modelo por medio del estadístico de Hosmer y Lemeshow. Los efectos de las variables 
predictoras sobre la aparición de carne PSE se expresaron por medio de las razones de dispariedad (OR) y sus respectivos intervalos de confianza del $95 \%$. En los diferentes análisis, todos los valores de $\mathrm{p}<0,05$ fueron considerados como significativos.

\section{RESULTADOS}

El transporte de los cerdos desde las granjas hasta la planta de beneficio fue de 1,4 h en promedio. El 44,2\% de los cerdos fue transportado en camiones de un piso y el 55,8\% restante, en camiones de dos pisos. Los vehículos, en general, cumplieron con los requisitos de la legislación sanitaria (9). Los pisos de los camiones eran antideslizantes y los vehículos estaban dotados de carpas de lona para proteger a los animales de las condiciones climáticas adversas. El 30,8\% de los transportadores realizó paradas durante el viaje (> $15 \mathrm{~min}$ ), en tanto que el $34,2 \%$ se detuvo solamente el tiempo necesario para hacer la inspección de los animales durante el viaje. Solo el 4,6\% de los lotes presentó mezcla de animales no familiares durante el transporte. La disponibilidad de espacio por animal exigida por la normatividad colombiana es de $1 \mathrm{~m}^{2}$ por animal en los corrales de recepción y sacrificio, aspecto que se cumplió en el presente estudio (9). El $72 \%$ de los cerdos fueron sometidos a periodos de ayuno entre 8 y 24 h en la granja y el restante entre 28 y $48 \mathrm{~h}$. El 25,1\% de los cerdos permaneció en la planta por un lapso superior a 24 h sin ser alimentados. La descripción de las variables continuas usadas en el análisis se presenta en la Tabla 1.

Tabla 1. Media, desviación estándar (DE), mínimo y máximo para las variables continúas usadas en el análisis.

\begin{tabular}{lcccc}
\hline \multicolumn{1}{c}{ Variable } & Media & DE & Mínimo & Máximo \\
\hline Densidad en el camión $\left(\mathrm{kg} / \mathrm{m}^{2}\right)$ & 217,4 & 72,9 & 45,4 & 428,8 \\
Disponibilidad de espacio en el camión $\left(\mathrm{m}^{2} /\right.$ & 0,6 & 0,2 & 0,3 & 4,5 \\
animal) & & & & \\
Peso promedio $(\mathrm{kg})$ & 110,2 & 20,3 & 66,7 & 246,7 \\
Velocidad promedio $(\mathrm{km} / \mathrm{h})$ & 56,0 & 10,1 & 20,0 & 70,0 \\
Tiempo de transporte $(\mathrm{h})$ & 1,4 & 1,3 & 0,2 & 6,0 \\
Densidad en corral $\left(\mathrm{kg} / \mathrm{m}^{2}\right)$ & 93,6 & 35,3 & 8,6 & 181,7 \\
Disponibilidad de espacio en el corral $\left(\mathrm{m}^{2} /\right.$ & 1,4 & 1,3 & 0,7 & 39,5 \\
animal) & & & & \\
Duración de la estadía en la planta de beneficio $(\mathrm{h})$ & 18,8 & 7,7 & 4,6 & 38,8 \\
Tiempo de ayuno en la granja (h) & 24,3 & 10,4 & 8,0 & 48,0 \\
Tiempo de ayuno total $(\mathrm{h})$ & 46,5 & 11,7 & 18 & 72,5 \\
\hline
\end{tabular}

El $82,9 \%(n=2.616)$ de los cerdos fue correctamente insensibilizado; solo el 17,1\% ( $\mathrm{n}=540)$ de los animales presentó signos de retorno a la consciencia. El signo más observado fue el intento de incorporarse $(60 \%)$, seguido de las vocalizaciones $(23,5 \%)$, el reflejo palpebral positivo $(14 \%)$ y la presencia de dolor $(2,5 \%)$.
El 100\% de las canales presentó contusiones cutáneas, con un promedio de 6,1 contusiones/ canal. La prevalencia de contusiones leves y severas fue $38,2 \%(n=1.207)$ y $61,8 \%$ ( $n=$ 1.949), respectivamente. Las contusiones más frecuentes estuvieron localizadas en el lomo $(32,0 \%)$ y el jamón (22,0\%). La caracterización de las contusiones cutáneas se presenta en Tabla 2. 
Marlyn Hellen Romero Peñuela, Jorge Alberto Sánchez Valencia

Tabla 2. Características de las contusiones cutáneas presentes en las canales evaluadas ( $n=19.175$ contusiones).

\begin{tabular}{|c|c|c|}
\hline Variable & $\mathbf{n}$ & $\%$ \\
\hline \multicolumn{3}{|l|}{ Localización } \\
\hline Cabeza & 2.081 & 11,0 \\
\hline Espalda & 3.573 & 19,0 \\
\hline Lomo & 6.219 & 32,0 \\
\hline Medio & 3.085 & 16,0 \\
\hline Jamón & 4.217 & 22,0 \\
\hline \multicolumn{3}{|l|}{ Forma } \\
\hline Coma & 13.763 & 71,8 \\
\hline Rectangular & 30 & 0,2 \\
\hline Lineal & 2.063 & 10,8 \\
\hline Difusa & 2.752 & 14,4 \\
\hline Marca de la cámara de $\mathrm{CO}_{2}$ & 567 & 3,0 \\
\hline \multicolumn{3}{|l|}{ Tamaño $(\mathrm{cm})$} \\
\hline $0,5-1$ & 435 & 2,3 \\
\hline $2-5$ & 6.817 & 35,6 \\
\hline $6-10$ & 4.095 & 21,4 \\
\hline $11-15$ & 2.460 & 12,8 \\
\hline $15<$ & 5.368 & 28,0 \\
\hline \multicolumn{3}{|l|}{ Concentración } \\
\hline Juntas & 12.792 & 66,7 \\
\hline Aisladas & 6.383 & 33,3 \\
\hline \multicolumn{3}{|l|}{ Color } \\
\hline Rojo & 17.158 & 97,2 \\
\hline Marrón & 488 & 2,8 \\
\hline
\end{tabular}

El 5,8\% (183/3.156) de las canales evaluadas presentó carne PSE. El análisis de regresión logística multivariada estableció que el tiempo de transporte, la densidad de carga en el camión, el tiempo de ayuno en granja y tiempo de ayuno total, las características del camión y la efectividad de la insensibilización, no estuvieron relacionadas con en la presencia de carne PSE en el presente estudio $(p>0,05)$. Las variables peso de llegada, el tiempo de estadía en la planta de beneficio y la presencia de contusiones cutáneas, son posibles factores de riesgo que incidieron en su presentación (Tabla 3). 
Tabla 3. Factores de riesgo para la presencia de carnes con $\mathrm{pH}_{45} \leq 5,8$ en porcinos comerciales evaluados por el análisis de regresión logística multivariada $(\mathrm{n}=3.156)$.

\begin{tabular}{lccccc}
\hline \multicolumn{1}{c}{ Variable } & Categoría & $\%$ & OR & ES & Valor p \\
\hline $\begin{array}{l}\text { Tiempo de estadía en } \\
\text { planta }(\mathrm{h})\end{array}$ & $4-10$ & 13,8 & 1,0 & & Ref. \\
& $10,1-12$ & 9,9 & 1,13 & 0,7 & 0,8 \\
& $12,1-18$ & 33,2 & 4,3 & 0,6 & $<0,01$ \\
& $18,1-24$ & 19,4 & 5,9 & 0,8 & $<0,01$ \\
& $24,1-36$ & 23,7 & 9,9 & 3,7 & $<0,01$ \\
& & & & & \\
Peso vivo (kg) & $80-120$ & 87,3 & 1,0 & & Ref. \\
& $121-150$ & 9,2 & 0,34 & 0,14 & $<0,01$ \\
& $151-200$ & 3,5 & 0,38 & 0,28 & 0,2 \\
Presencia de contusiones & & & & & Ref. \\
cutáneas & Sí & 78 & 1,0 & & $<0,01$ \\
\hline
\end{tabular}

* OR: razones de disparidad. Ref.: categoría considerada como referencia.

De acuerdo con los resultados obtenidos en el análisis de regresión logística multivariada (Tabla 3), se elaboraron las figuras de la función de la probabilidad de presentar carne PSE, frente a las variables que fueron significativas en el análisis. El peso de llegada de los cerdos a la planta presentó una relación inversa con la prevalencia de carne PSE, es decir que a mayor peso, disminuyó la probabilidad de ésta (Figura 2). El tiempo de permanencia en planta presentó una relación directa, aumentando la probabilidad de carne PSE, a medida que se incrementaba el tiempo (Figura 3).

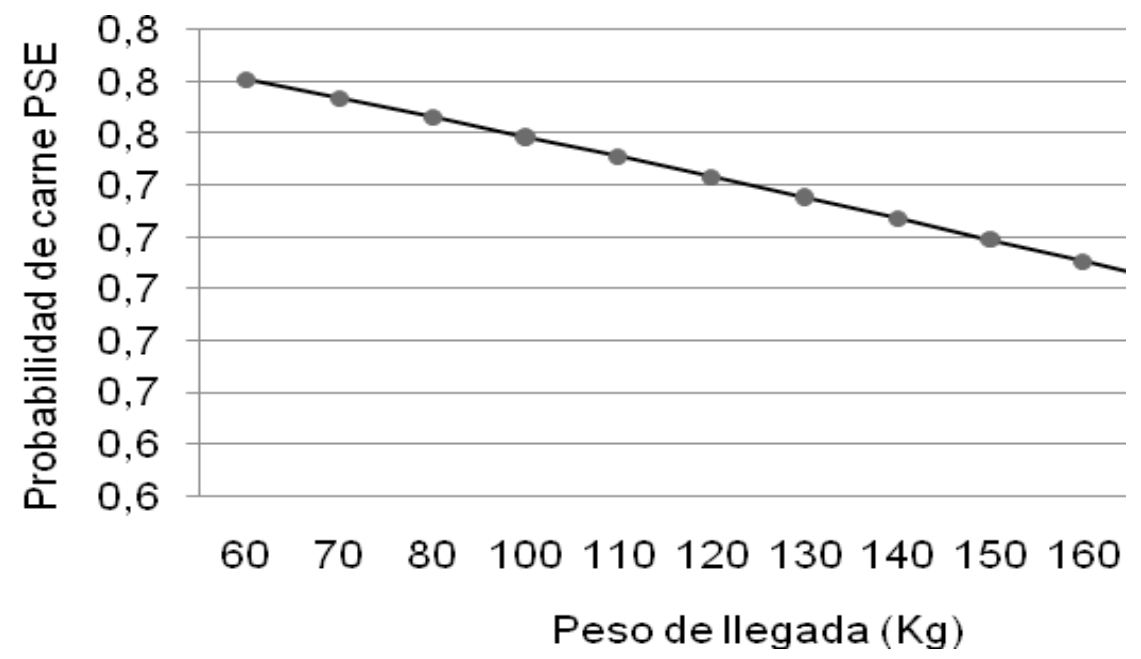

Figura 2. Cálculo de probabilidad de la presencia de carne PSE en función del peso de llegada de los cerdos a la planta de beneficio. 


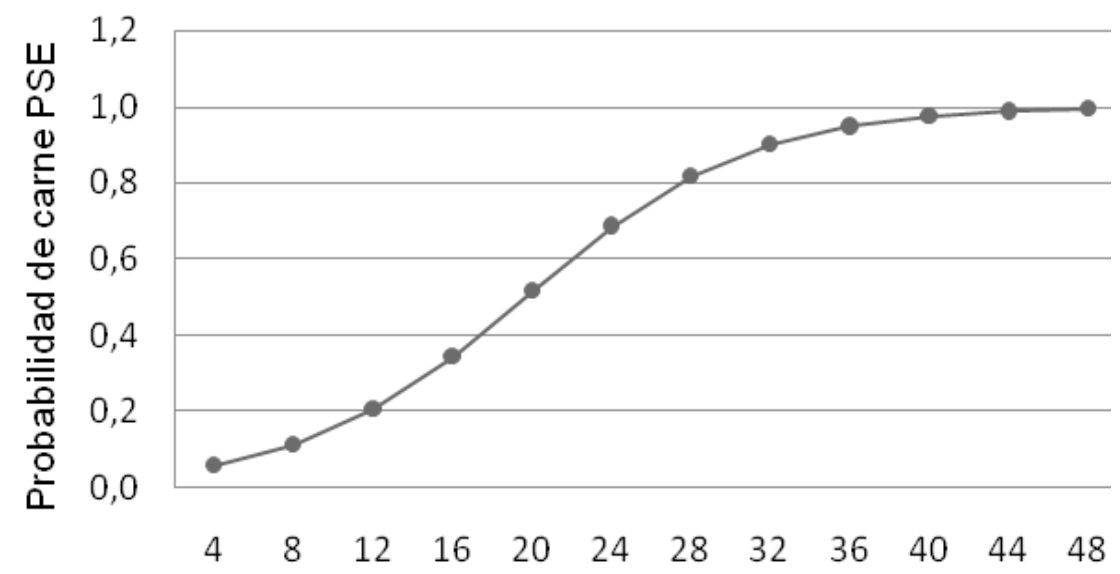

Tiempo de estadía en planta $(\mathrm{h})$

Figura 3. Cálculo de probabilidad de la presencia de carne PSE en función de la estadía en la planta de beneficio.

\section{DISCUSIÓN}

La prevalencia de carne PSE en el presente trabajo fue baja, resultados discordantes con los descritos en Colombia (2), los cuales han reportado valores del 25,2\%; sin embargo, no se pueden efectuar comparaciones absolutas porque la calidad de la carne es una variable multifactorial que depende de las características genéticas de los animales, el manejo presacrificio, las condiciones ambientales y la velocidad de glucólisis, entre otros aspectos (10).

El transporte es una de las etapas críticas para el Bienestar Animal (BA) y la calidad de la carne (12). Sin embargo, en el estudio, el tiempo de transporte fue de corta duración porque las granjas hacían parte de una cooperativa de productores, se encontraban localizadas en el área de influencia de la planta de beneficio y los camiones utilizados para el transporte presentaron buenas condiciones de mantenimiento, aspectos que en conjunto pudieron incidir en la no asociación entre esta variable y la presencia de carne PSE. Asi mismo, estuvo acorde con los criterios exigidos por la legislación sanitaria, que establece que la duración del transporte de los cerdos, no debe ser mayor a 8 h (9). El tipo de camión utilizado para transportar los cerdos ha sido considerado como un factor de riesgo para la presencia de contusiones y de mortalidad durante el transporte $(10,13)$. En el presente estudio, el tipo de camión no se asoció con la presentación de carne PSE.

Las densidades de carga en el camión afectan el comportamiento porcino, el bienestar, la calidad de la carne, tienen un efecto aditivo en el estrés presacrificio y cuando no son apropiadas, aumentan las pérdidas totales durante el transporte $(13,14)$. En el estudio se observó que las densidades de carga en los camiones no estaban estandarizadas y que se transportan animales tanto a altas, como a bajas densidades. El 63\% de los camiones cumplieron con las densidades de carga exigidas por la normatividad sanitaria colombiana $\left(0,5 \mathrm{~m}^{2} /\right.$ animal para cerdos de $100 \mathrm{~kg}$ de peso y $0,8 \mathrm{~m}^{2} /$ animal para animales adultos) (9). Densidades muy altas generan gastos de energía adicionales, porque aumentan los encuentros antagónicos entre los cerdos y dificultan la posibilidad de descanso, lo cual se traduce en alteraciones del pH en la carne (6). Las bajas densidades tienen implicaciones en la calidad de la carne, porque 
los cerdos al presentar una mayor disponibilidad de espacio en el camión tienen dificultad para mantener el equilibrio, lo que favorece las caídas, las colisiones contra la carrocería del camión y el gasto de energía adicional (15). Esta variable no estuvo relacionada con la presencia de carne PSE, como sí se reportó en Colombia durante el transporte de bovinos (16) y en otros estudios en Norte América, donde la densidad de carga se ha asociado a pérdidas por lesiones y la aparición de carne porcina de menor calidad (13). Sin embargo, esta falta de asociación fue concordante con otros estudios en porcinos, donde se reporta que densidades de $0,4 \mathrm{~m}^{2} / 100$ $\mathrm{kg}$, muy similares a las observadas en Colombia, tienen un efecto muy bajo sobre la calidad de la carne, en especial en viajes de corta duración (< 3 h) (17), característica observada en este estudio.

El periodo de ayuno de los cerdos previo al transporte hacia las plantas de beneficio, no está reglamentado en Colombia, por lo que los productores seleccionan sus propios criterios (9). La normatividad sanitaria establece que los cerdos deben permanecer en la planta de beneficio mínimo $4 \mathrm{~h}$, para favorecer el descanso y la inspección sanitaria antemortem; sin embargo, este período no puede exceder las 48 h (8). La falta de asociación entre el tiempo de ayuno y la presencia de carne PSE puede estar relacionada con el hecho de que la mayor proporción de animales tuvo períodos de ayuno entre 8 y $24 \mathrm{~h}$, los cuales son considerados como una buena práctica para prepararlos para el transporte (3). No obstante, tiempos adicionales de ayuno pueden incrementar la agresividad de los cerdos y tiempos inferiores a $12 \mathrm{~h}$ pueden aumentar el riesgo de encontrar carnes próximas a los valores de $\mathrm{pH}$ considerados como PSE (6). Otros autores han inferido que el ayuno porcino produce un agotamiento del glucógeno muscular y carnes con $\mathrm{pH}$ musculares altos, característicos de la condición DFD (18).

La duración y las condiciones de la estadía en la planta de beneficio (características de las instalaciones, disponibilidad de espacio en el corral, manejo, condiciones ambientales, etc.) tienen gran impacto en el BA y la calidad de la carne $(17,19)$. Unas condiciones adecuadas permiten la recuperación de los cerdos de manejos y factores estresantes previos (15). La planta de beneficio estudiada contaba con infraestructura moderna, basada en criterios de BA, suministro de agua a voluntad y cumplía con todos los requerimientos sanitarios previstos en la legislación sanitaria (8). A pesar de esto, se apreció que los tiempos de estadía en planta superiores a las $12 \mathrm{~h}$ incrementaron el riesgo de carnes PSE debido probablemente al desafío de los animales con situaciones no familiares como los ambientes nuevos de la planta de beneficio, el ruido, la actividad física y el contacto con personal extraño, factores que generan estrés y pueden incidir en el metabolismo muscular previo al sacrificio, aspecto descrito por otros autores $(20,21)$. Adicionalmente, ha sido evidente que duraciones de estadía en planta inferiores a las observadas en esta investigación, disminuyen la probabilidad de encontrar carnes de menor calidad $(6,20)$.

La insensibilización inefectiva no fue un factor de riesgo para la presentación de carne PSE en el estudio; estos mismos hallazgos fueron reportados por investigadores que compararon diferentes sistemas de aturdido y el pH de la carne porcina (22) y en cerdos insensibilizados eléctricamente (15). A pesar de que la insensibilización fue efectiva en la mayor proporción de los cerdos del estudio, es necesario prestar atención a los signos de pérdida de la consciencia más frecuentes, como fue el intento de incorporarse, signo correspondiente al "reflejo de enderezamiento", que hace parte de las reacciones posturales indicadoras de alteraciones nerviosas en la transmisión de señales al mesencéfalo, el cual es considerado como un signo confiable y último de retorno completo de la consciencia (23), por lo cual su presencia no puede ser aceptada bajo ninguna circunstancia en la banda de sangría sin una acción correctiva, aspecto que no se observó en la planta estudiada. Aunque la respuesta al dolor 
y las vocalizaciones probablemente no indican el estado de consciencia del animal, una reacción positiva puede relacionarse con la posibilidad eventual del retorno a la consciencia (23).

Las contusiones en la piel han sido utilizadas como indicadores de $\mathrm{BA}$ en el presacrificio porcino $(10,15)$. La categorización de las contusiones permitió establecer que la mayor proporción de éstas fueron consideradas como severas y se localizaron principalmente en el jamón y el lomo, que son las áreas más expuestas del cerdo, en donde se ubican las lesiones que son infligidas por los operarios durante la movilización, lo que evidencia problemas de la relación hombre-animal y de BA, que producen sufrimiento en este proceso (24). Además, fueron frecuentes las contusiones múltiples en forma de coma, concentradas en diferentes áreas y con tamaños entre 2 y $10 \mathrm{~cm}$, características de las producidas por mordeduras durante los encuentros antagónicos de los cerdos, por problemas de hacinamiento o mezcla de animales no familiares (25). De otra parte, predominaron las contusiones recientes o rojas que indican que la mayoría de éstas se infligieron en la planta, durante las $10 \mathrm{~h}$ previas al sacrificio, es decir, durante la estadía en los corrales y la conducción hacia el área de insensibilización (11). Los cerdos que presentaron contusiones cutáneas leves tuvieron menor probabilidad de presentar carnes PSE; similares resultados obtuvieron investigadores en España, quienes encontraron que a mayor severidad de las contusiones, menor calidad instrumental de la carne y niveles más altos de cortisol sanguíneo, indicativo de estrés fisiológico (5). Estos resultados sugieren que las prácticas de manejo cruentas y los encuentros antagónicos entre los animales durante el presacrificio, generadores de contusiones cutáneas, son factores que favorecen la presencia de estrés agudo en los cerdos, inmediatamente antes del sacrificio, lo que causa un incremento en la tasa de acidificación postmortem y la presencia de la condición PSE (16).
Respecto a la relación entre el peso vivo de los animales y la aparición de la condición PSE, en el estudio los animales con pesos entre 80 y $120 \mathrm{~kg}$ fueron los más frecuentes, porque corresponden a las características fenotípicas requeridas comercialmente y provienen de granjas con programas de mejoramiento genético, motivo por el cual tal vez debido a su mayor conformación muscular, menor contenido de grasa dorsal y que provienen generalmente de líneas genéticas más susceptibles al estrés, tuvieron mayor riesgo de presentar carne PSE, que los animales con mayor peso, que corresponden a animales de descarte (26). No obstante, en futuras investigaciones sería conveniente conocer las condiciones genéticas de los cerdos evaluados para establecer si son portadores del gen halotano, que los hace más susceptibles al estrés; así como la evaluación del grosor del tejido muscular en las canales.

\section{CONCLUSIONES}

Los cortos tiempos de transporte, las densidades de carga en el camión, la efectividad de la insensibilización y el ayuno, no estuvieron asociados con la presencia de carne PSE. El tiempo de estadía en la planta de beneficio superior a $12 \mathrm{~h}$ favoreció la glicólisis muscular y la disminución del pH de la carne en los cerdos evaluados, probablemente debido al desafío de los animales con situaciones no familiares como los ambientes nuevos de la planta de beneficio, el ruido y el contacto con personal extraño. La carne obtenida de cerdos que tuvieron contusiones cutáneas leves presentó valores de $\mathrm{pH}$ relacionados con parámetros de mejor calidad. Los cerdos con pesos entre 80 y $120 \mathrm{~kg}$ tuvieron mayor riesgo de presentar carne PSE al compararse con los animales de mayor peso. Los resultados muestran que el efecto del manejo presacrificio sobre la aparición de la condición PSE es un factor multicausal que amerita mayor investigación, especialmente cuando se trabaja bajo condiciones comerciales. 


\section{REFERENCIAS}

1. Departamento Nacional de Planeación, Consejo Nacional de Política Económica y Social. Documento CONPES 3458, de Política Nacional de sanidad e inocuidad para la Cadena porcícola. Enero 29 de 2007.

2. Castrillón WE, Fernández JA, Restrepo LF. Variables asociadas con la presentación de carne PSE (Pálida, Suave, Exudativa) en canales de cerdo. Rev Colom Cienc Pecua 2007; 20:327-338.

3. Barbut S, Sosnicki AA, Lonergan SM, Knapp T, Ciobanu DC, Gatcliffe LJ, et al. Progress in reducing the pale, soft and exudative (PSE) problem in pork and poultry meat. Meat Sci 2008; 79:46-63.

4. O'Neil DJ, Lynchb PB, Troyc DJ, Bluckleya DJ, Kerrya JP. Influence of the time of the year on the incidence of PSE meat and DFD in Irish pig meat. Meat Sci 2003; 64:105-111.

5. Gispert M, Faucitano L, Oliver MA, Guàrdia MD, Coll A, Siggens K, et al. A survey of pre-slaughter conditions, halothane gene frequency, and carcass and meat quality in five Spanish pig commercial abattoirs. Meat Sci 2000; 55:97-106.

6. Guàrdia MD, Estany J, Balasch S, Oliver MA, Gisper MT, Diestre A. Risk assessment of skin damage due to pre-slaughter conditions and RYR1 gene in pigs. Meat Sci 2009; 81:745-751.

7. Decreto 1500 de 2007, Reglamento técnico del Sistema Oficial de Inspección, Vigilancia y Control de la Carne, Productos Cárnicos Comestibles y Derivados cárnicos, requisitos sanitarios y de inocuidad que se deben cumplir en su producción primaria, beneficio, desposte, desprese, procesamiento, almacenamiento, transporte, comercialización, expendio, importación o exportación. Boletín Oficial del Estado, número 46.618. Mayo 4 de 2007.

8. Resolución 00240, Requisitos sanitarios para el funcionamiento de las plantas de beneficio animal de las especies bovina, bufalina y porcina. Boletín Oficial del Estado, número 48.699. Febrero 9 de 2013.

9. Resolución 2640 de 2007 , Condiciones sanitarias y de inocuidad en la producción primaria de ganado porcino destinado al sacrificio para consumo humano. Boletín Oficial del Estado, número 46.768. Octubre 1 de 2007.

10. Dalla Costa OA, Faucitano L, Coldebell A, Ludke JV, Peloso JV, Roza D, Paranhos da Costa MJR. Effects of the season of the year, truck type and location on truck on skin bruises and meat quality in pigs. Lives Sci 2007; 107(1):29-36.

11. Varón-Álvarez LJ, Romero MH, Sánchez JA. Caracterización de las contusiones e identificación de factores de riesgo durante el manejo presacrificio de cerdos comerciales. Arch Med Vet 2014; 46:151159.

12. Frisby J, Raftery D, Kerry JP, Diamond D. Development of an autonomous, wireless $\mathrm{pH}$ and temperature sensing system for monitoring pig meat quality. Meat Sci 2005; 70:329-336.

13. Fitzgerald RF, Stalder KJ, Matthews JO, Schultz Kaster CM, Johnson AK. Factors associated with fatigued, injured, and dead pig frequency during transport and lairage at a commercial abattoir. J Anim Sci 2009; 87:1156-1166.

14. Ritter MJ, Ellis M, Brinkmann J, DeDecker JM, Keffaber KK, Kocher ME, et al. Effect of floor space during transport of market-weight pigs on the incidence of transport losses at the packing plant and the relationships between transport conditions and losses. J Anim Sci 2006; 84:2856-2864.

15. Van de Perre V, Permentier L, De Bie S, Verbeke G, Geers R. Effect of unloading, lairage, pig handling, stunning and season on pH of pork. Meat Sci 2010; 86:931-937.

16. Romero MH, Uribe-Velásquez LF, Sánchez JA, Miranda de-la Lama G. Risk factors influencing bruising and high muscle $\mathrm{pH}$ in Colombian cattle carcasses due to transport and pre-slaughter operations. Meat Sci 2013; 95:256-263.

17. Warriss PD, Brown SN, Knowles TG, Edwards JE, Kettlewell PJ, Guise HJ. The effect of stocking density in transit on the carcass quality and welfare of slaughter pigs: 2 . Results from the analysis of blood and meat samples. Meat Sci 1998; 50(4):447-456. 
18. Leheska JM, Wulf DM, Maddock RJ. Effects of fasting and transportation on pork quality development and extend of postmortem metabolism. J Anim Sci 2003; 81:3194-3202.

19. Hambrecht E, Eissen JJ, Newman DJ, Smits CHM, den Hartog LA, Verstegen MWA. Negative effects of stress immediately before slaughter on pork quality are aggravated by suboptimal transport and lairage conditions, J Anim Sci 2005; 83:440-448.

20. Nanni Costa L, Lo Fiego DP, Dall'Olio S, Davoli R, Russo V. Combined effects of pre-slaughter treatments and lairage time on carcass and meat quality in pigs with different halothane genotypes. Meat Sci $2002 ; 61: 41-47$.

21. Zhen S, Liu Y, Li X, Ge K, Chen H, Li C, Ren F. Effects of lairage time on welfare indicators, energy metabolism and meat quality of pigs in Beijing. Meat Sci 2013; 93:287-291.

22. Channon HA, Payne AM, Warner RD. Comparison of $\mathrm{CO}_{2}$ with manual electrical stunning $(50 \mathrm{~Hz})$ of pigs on carcass and meat quality. Meat Sci 2002; 60:63-68.

23. European Food Safety Authority. Welfare aspects of the main systems of stunning and killing the main commercial species of animals. EFSA J 2004; 45:1-29.

24. Correa JA, Torrey S, Devillers N, Laforest JP, Gonyou HW, Faucitano L. Effects of different moving devices at loading on stress response and meat quality in pigs. J Anim Sci 2010; 88:4086-4093.

25. Turner SP, Farnworth JM, White IMS, Brotherstone S, Mendl M, Knap P, et al. The accumulation of skin lesions and their use as a predictor of individual aggressiveness in pigs. Appli Anim Behav Sci 2006; 96:245-259.

26. Alarcón-Rojo AD, Gamboa-Alvarado JG, Rodríguez-Alameida FA, Grado-Ahuir JA, Janacua-Vidales H. Efecto de variables críticas del sacrificio sobre las propiedades fisicoquímicas de la carne de cerdo. Rev Mex de Cienc Pecuarias 2006; 44(1):53-66. 